munity. They are introducing this new software technology to fill an immediate and real need. Present-generation particle-accelerators produce such huge volumes of measurement data, at such high rates, that a new approach is needed to enable the information to be processed, analyzed, and interpreted by thousands of collaborating scientists throughout the world. To quote from the U.K.-funded GRIDPP Web site (http://www.gridpp.ac.uk):

The next IT revolution will be the Grid. While the Web is aimed mainly at the exchange of information, the Grid is concerned with the exchange of computer power, data storage, and access to large databases, without users searching for these resources manually. Computer services will become a utility like electricity, supplied via a Grid. Once connected to the Grid, the end user will see it essentially as one large computer system. The Grid is a practical solution to the data-intensive problems that must be overcome if the computing needs of many scientific communities and industry are to be fulfilled over the next decade.

Can future power networks benefit as a spin-off application? In power systems, as a result of the move to renewables, a larger number of smaller generators will call for more active and dynamic operation, especially at the distribution level. The future power system will involve many participants: generator owner/ operators, generator maintenance providers, generation aggregators, transmission network operators (TNOs), distribution network operators (DNOs), load managers, energy market makers, energy supply companies, metering companies, energy customers, regulators, and governments. Grid computing could offer an inexpensive and efficient means for participants to compete (but also cooperate) in providing reliable, cheap, and sustainable electrical energy supply.

\section{The Nature of Grid Computing}

Grid computing, or "the Grid" for short, uses standard open-source (nonpropriety) software to allow a large number of users to share computational resources. In contrast to the limited information sharing provided by the Web, all types of computational resources can be shared (i.e., data stores, algorithms, and processing power). The sharing of resources is by no means a "free-for-all," however. Access to shared resources is carefully controlled.
The computers participating in a grid create a massive distributed system, and the users become part of a "virtual organization.”

\section{Grid Computing and Distributed Computing}

In the early days of computer networking, various specialized systems enabled file transfer and e-mail within universities and research-led organizations. The introduction of the Web allowed that technology to reach critical mass and develop into the system that we are familiar with today. In a similar way, grid computing culminates from earlier developments in "distributed computing" and is widely predicted to be the "next big thing" in computing.

\section{Grid Computing and the Web}

Like the Web, the grid can operate over the Internet or any other suitable computer networking technology. How does the grid differ from the Web? Some of the significant differences are shown in Table 1.

\section{Characteristics and Capabilities}

Some of the relevant characteristics and capabilities of grid computing are as follows:

$\checkmark$ A grid is created by installing software services, or middleware [e.g., the Globus Toolkit (http://www. globus.org)], on a set of networked computers. The middleware provides facilities such as hardware and

\begin{tabular}{|ll|}
\hline \multicolumn{2}{|c|}{ table 1. Differences between the "Web" and the "Grid." } \\
\hline The "Web" & The "Grid" \\
\hline low-volume information sharing & $\begin{array}{l}\text { high-volume information sharing, distributed } \\
\text { computational processes, and computer } \\
\text { power sharing }\end{array}$ \\
there is only one Web & $\begin{array}{l}\text { there are many separate and distinct grids } \\
\text { anyone can "join" }\end{array}$ \\
$\begin{array}{l}\text { no intrinsic reliability and security } \\
\text { of data transmission }\end{array}$ & $\begin{array}{l}\text { registered participants } \\
\text { reliable and secure data transmission }\end{array}$ \\
no inherent protection against misuse & certification, authentication, etc., is built in
\end{tabular}




\section{Grid computing could offer an inexpensive and efficient means for participants to compete (but also cooperate) in providing reliable, cheap, and sustainable electrical energy supply.}

\section{An Example of a Developing Transnational Grid Application}

The large-scale adoption of grid computing has been motivated by a number of primarily scientific applications. As an example, and one that we contribute to at Brunel University, we outline briefly the grid being used by a large-scale internationally distributed collaborative experiment in the field of fundamental particle physics. Figure 1 shows the Compact Muon Solenoid Experiment (http://cmsinfo.cern.ch/Welcome.html/), which will operate at the "Large Hadron Collider" for a decade commencing in 2007 and will produce stored data at a rate of order 1 Pbyte per year (actual real-time data rate is about 106 times higher).
Figure 2 shows the structure of the GridPP (http://www.gridpp.ac.uk/) grid. The physicist (Tier-4 level) will access local (Tier-3) resources, analysis cluster, and local storage via a portal device (e.g., workstation, laptop, or hand-held). Tier-3 resources are connected to regional Tier- 2 centers that are able to service requests, for example, for Monte Carlo production. National Tier-1 centers are repositories for large quantities of data from the experimental sites (located at Tier- 0 centers). The hierarchical structure is aimed at minimizing bandwidth requirements, but the grid nature allows peer-to-peer communication and direct access to all facilities (broken lines) where authorization is approved.

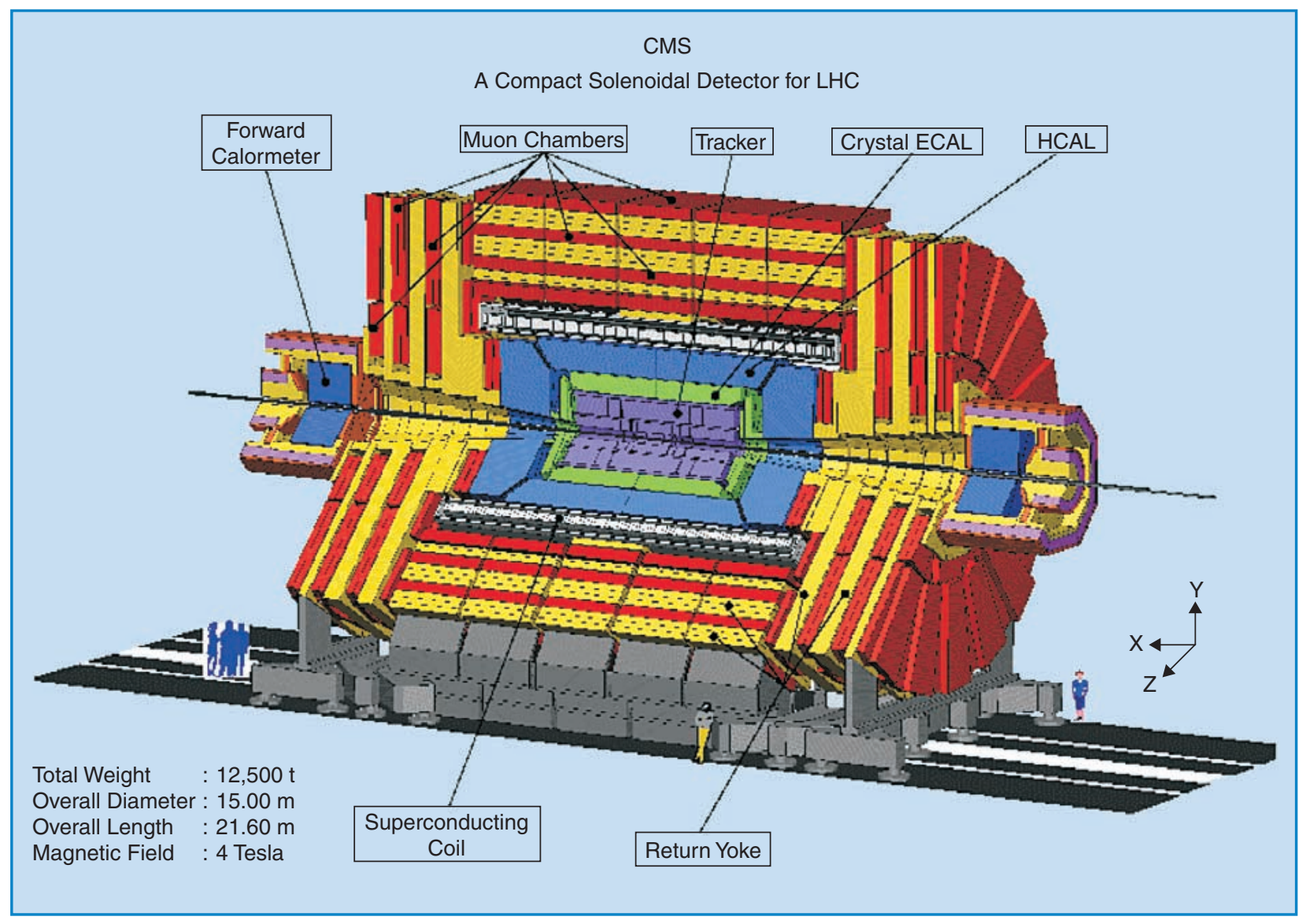

figure 1. The Compact Muon Solenoid (CMS) experiment currently under construction. This 12,500 ton particle detector operates at a rate of 40 million beam collisions per second and will produce stored data at a rate of order 1 Pbyte per year of operation. 


\section{Grid computing can provide a relatively inexpensive new technology allowing the output of embedded generators to be monitored and, when necessary, controlled.}

The organization of the implementation of the grid will take two forms: "clusters" and WorkGroups. In the United Kingdom there will be four regional clusters representing universities and institutes. These local groupings will collaborate to create regional centers of excellence for grid activities and build geographically localized regional Tier-2 centers that typically share a single MAN. The WorkGroups, whose remit closely match those within the EU DataGrid (http://eu-datagrid.web.cern.ch/eudatagrid/), are delivering the software components of the grid. Although in some WorkGroups there will be a strong regional interest, institutes within a cluster will collaborate with groups in other clusters. Thus, the implementation of the grid conforms to paradigm of "virtual organizations" through the creation of geographically intersecting WorkGroups and clusters that share resources.

Although the grid outlined above is primarily aimed at providing computational power and distributed data brokerage, plans are also underway to use the grid for remote monitoring and control of the CMS experimental apparatus itself.

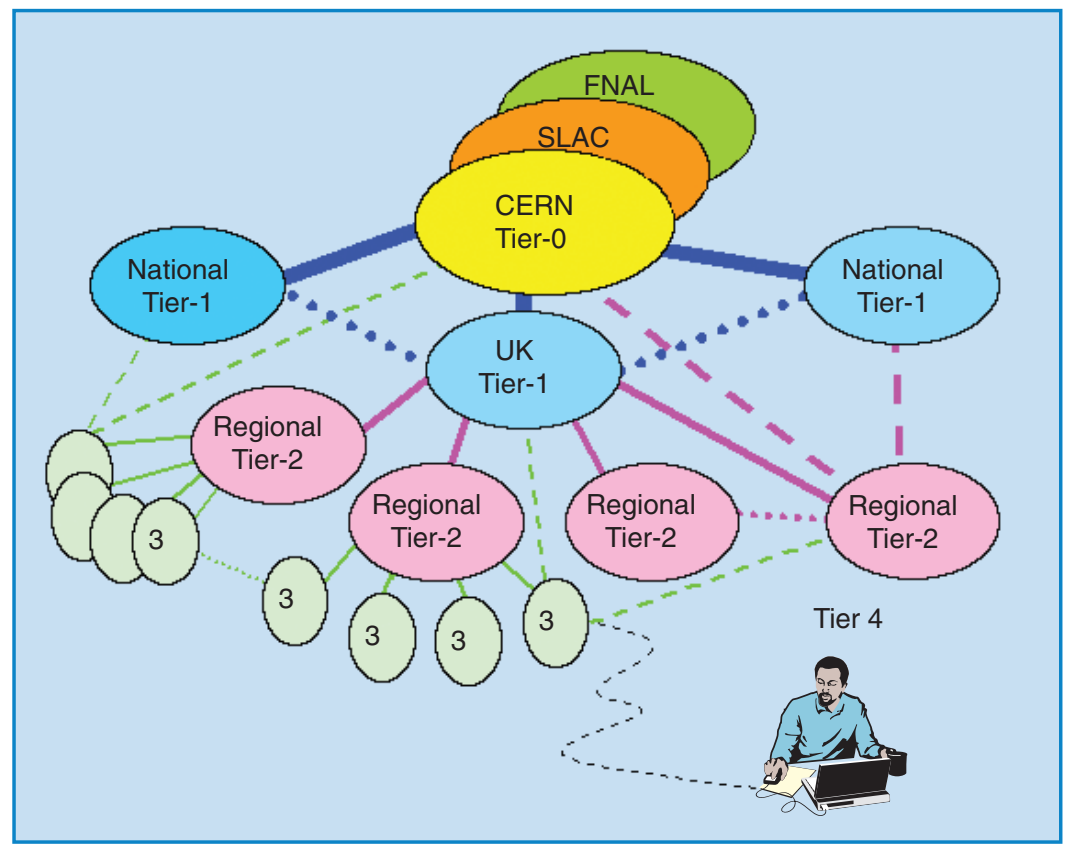

figure 2. The tiered structure of the particle physics grid "GRIDPP" for the Large Hadron Collider experiments. The physical experiment CMS is located at the CERN Tier-0 center in Switzerland, and in the U.K. the Tier-1 center is at CLRC RAL. Geographically distributed Tier-2 centers are located in Scotland, Northwest England, London, and Southwest England. Brunel University is a member of the "London" Tier 2 center and is also a local Tier-3 center in its own right.

\section{Potential Benefits for Future Power Systems}

Grid computing could have very significant benefits for almost all aspects of power systems that involve computers. In the following discussion, it is assumed that in the future, every electrical generator will be equipped with computational and communication facilities. This does not seem too farfetched in a world where cars, vending machines, domestic appliances, and so on are predicted to be similarly equipped.

\section{Monitoring and Control}

It is anticipated that a large number of smaller generators will be introduced in the coming decades, using renewable energy and reducing carbon emissions. As these generators are connected into the power system (often embedded at the distribution level), it will become necessary to monitor and control their

output level and their on/off schedule. The control technology in use at the transmission level is not scalable to very large numbers of generators. Grid computing can provide a relatively inexpensive new technology allowing the output of embedded generators to be monitored and, when necessary, controlled.

An outline of a power-systems grid-computing structure is illustrated in Figure 3.

\section{Market Entry and Participation}

Future electrical energy markets should provide open access to a range of participants from very small organizations (perhaps even individuals) to very large companies. The need to register in the market, receive and transmit financial information, notify the market of technical constraints, and so on can all be provided by grid computing. The ability of grid-enabled systems to interact autonomously will be vital for small generators where manned operation is unlikely to be viable. 


\section{The ability of grid-enabled systems to interact autonomously will be vital for small generators where manned operation is unlikely to be viable.}

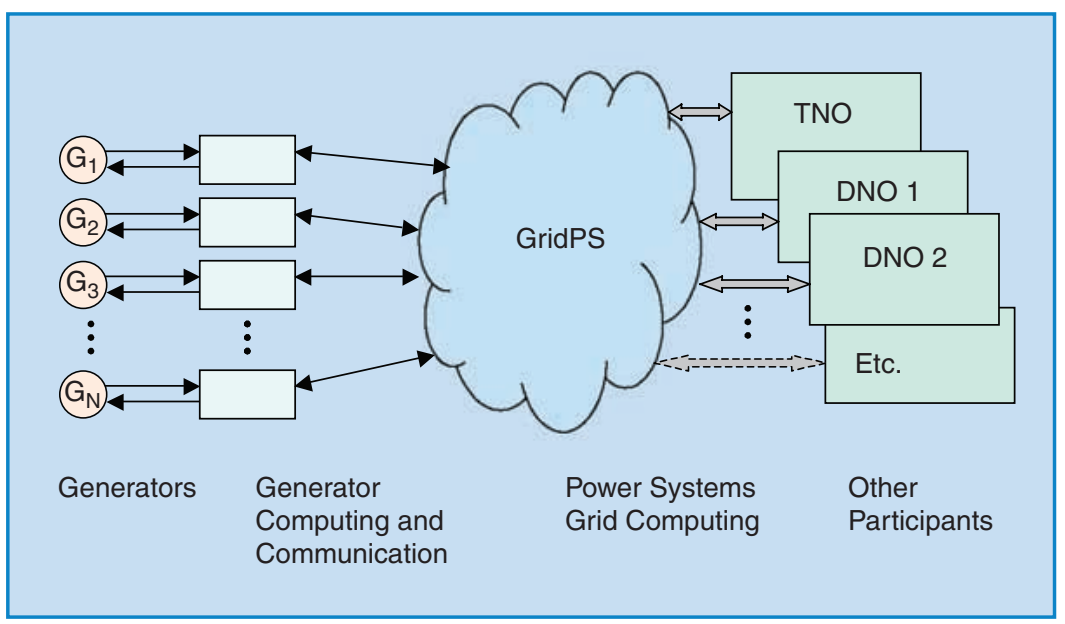

figure 3. Outline of a power-systems computing grid.
States. Major computer manufacturers are already seriously committed. However, to our knowledge, there are no projects underway to investigate power system applications. At Brunel University, power system and grid-computing research teams are collaborating and have obtained funding to underpin research in this area. It is hoped that this field will develop rapidly over the next few years, bringing forward a new technology with significant benefits for the power networks of the future.

The authors would be happy to be contacted by anyone interested in taking the discussion further. See the For Further Reading section at the end of this article for two excellent introductions to grid

\section{Regulation}

A power-system computing grid would provide regulators with access to complete information on the technical and economic performance of regulated markets. New regulatory rules and incentives could be introduced and disseminated electronically.

\section{Planning}

Planning of future power systems will rely on a collective effort by many companies and agencies. The sharing of accurate, detailed information on the existing system and the ability to use industry-wide computer models and forecasts would facilitate a coordinated approach. The creation of a "virtual organization," including all relevant participants, could retrieve some of the benefits of global planning that are often claimed to have been the main advantage of the centralized, vertically integrated industry that existed before privatization.

\section{Further Development}

More than 20 projects are already underway to develop and exploit grid computing. The U.K. research effort includes a $£ 98$ million initiative, established by the Office of Science and Technology. A National e-Science center has been created with a number of regional centers (http://www.nesc.ac.uk). Considerably greater funding has been made available in the European Union and the United computing technology.

\section{For Further Reading}

I. Foster and C. Kesselman, Eds., The Grid: Blueprint for a New Computing Infrastructure. San Mateo, CA: Morgan Kaufmann, 1999.

F. Berman, G. Fox, and A.J.G. Hey, Grid Computing: Making the Global Infrastructure a Reality. New York: Wiley, 2003.

\section{Biographies}

Malcolm Irving is professor of power systems and director of the Brunel Institute of Power Systems at Brunel University, London, United Kingdom. He has published many research papers on computer methods and algorithms for power system analysis and operation. He is a Member of the IEEE.

Gareth Taylor is a lecturer within the Department of Electronic and Computer Engineering, Brunel University, London, United Kingdom. He has published an extensive range of papers concerning applied mathematical modelling

Peter Hobson is a reader in the Sensors Instrumentation and Radiation Effects group at Brunel University, London, United Kingdom. He is a particle physicist currently working on vacuum photo-detectors and grid computing for the Compact Muon Solenoid experiment. and power system analysis. 